\title{
Effective Integration of Teaching and Research in a First Year Food and Nutrition Course
}

\author{
Russell Keast, Susie Macfarlane, Lynn Riddell \\ School of Exercise and Nutrition Sciences, Deakin University, Victoria, Australia \\ Email: Russell.keast@deakin.edu.au
}

Received October $2^{\text {nd }}, 2012$; revised November $4^{\text {th }}$, 2012; accepted November $20^{\text {th }}, 2012$

\begin{abstract}
Previous research has indicated that undergraduate student learning can be enhanced through active involvement in research. Furthermore, creating an academic environment where teaching and research are intimately linked can facilitate the induction of students into a community of learners where new knowledge is created, explored and critiqued. Scaffolding and supporting student learning via engagement in authentic research experiences can work to ensure graduating students have the capacity to generate and investigate important questions that contributes to the development of new knowledge. This paper presents a case study that outlines curriculum design and pedagogical strategies aimed at integrating teaching and research within the first year of an undergraduate course. First year Food and Nutrition students were asked to partake in a research project where they were asked to complete a series of diet and food related questionnaires, analyse, interpret and critique the resulting data. Students were supported through this learning activity via small group tutorial support and question and answer sessions within the learning management system. Anonymous evaluation of the teaching and learning experience was conducted at the end of the teaching period and the results indicate that the students welcomed the opportunity to engage in an authentic, research based learning activity. Students' found the assessment tasks were clearly explained to them (88\% agreeing), and felt well supported in approaching this research based assessment task. Furthermore, the qualitative comments indicated that the students' found the learning environment to be meaningful and relevant. This case study indicates that it is possible to effectively incorporate authentic research experiences within the curriculum of a first year course. The experiential, inquiry based learning approach used supported the students' participation in a systematic, rigorous data collection process required in a structured research environment and blended these requirements with authentic learning of discipline specific skills and knowledge.
\end{abstract}

Keywords: Higher Education; Teaching Research Nexus; Student Experience

\section{Introduction}

\section{The Teaching-Research Nexus}

The teaching-research nexus is a multi-faceted notion that holds various meanings and encompasses a range of activities centred on the interdependence between teaching and research. These activities are enacted at various sites of the university: 1) the research conducted by the teaching staff that forms part of the content of the course. The students' role here is to act as an "audience to the research carried out by their [teachers]" (Zamorski, 2002); 2) the teaching and learning activities that include research conducted with and by undergraduate and postgraduate students; 3 ) teaching staff involvement in the scholarship of teaching and learning (Prince, Felder, \& Brent, 2007). The pressure on universities to increase research output, identify and market their distinctive approaches to teaching and meet community demand for graduates who are prepared for challenging roles in complex workplaces has to lead to a growing focus on enhancing the teaching-research nexus (Zubrick, Reid, \& Rossiter, 2001).

\section{Impact of Research on Teaching Quality}

The findings regarding the impact of research on teaching quality indicate that at both an institutional level and that of an individual academic, research productivity has no impact on, and in some cases reduces teaching performance. At the level of the institution, a longitudinal study by Astin (as cited in Prince et al., 2007) examining the impact of research on educational outcomes found a significant negative correlation. Students attending more research-oriented institutions experience higher levels of dissatisfaction and poorer results in most measures of cognitive and affective development. This finding may be explained by a lack of alignment between research and teaching activities at an institutional level. Jenkins (2004) observes that within universities, research and teaching are managed separately, and that policy changes are required to integrate research and teaching goals and activities.

Several reviews undertaken of studies on the relationship between the level of research activity conducted by an academic and their teaching effectiveness find no relationship (Feldman, 1987; Hattie \& Marsh, 1996; Jenkins, 2004). Research excellence and productivity is independent of teaching excellence: some academics are highly productive researchers and skilled teachers, some have mastery in one area and not the other, and others are weak in both. Two explanations for this finding have been proposed. One explanation relates to the institutional context: that as each activity requires an almost full-time workload, the significant demands on academics' time necessitate the de- 
cision to focus primarily on either research or teaching (Prince et al., 2007).

A second explanation for the lack of association between research and teaching excellence is that the skills and attributes required to undertake rigorous research are very different to those required to excel as a university teacher. For example, Coaldrake and Stedman (1999) propose the dichotomy that research is the generation of new knowledge while teaching is the transmission of knowledge. However, effective teaching is not a process of transmission, but rather the facilitation of others' learning and discovery. Traditional teaching approaches in which students attend lectures in which they are expected to passively absorb information then apply this transmitted knowledge through essay or examination have little in common with the research process involving developing hypotheses to test open-ended problems characterised by complex, missing or conflicting information, and producing and defending final results (Prince et al., 2007). It is therefore unsurprising that teaching approaches consisting of a one-way communication of research findings results has little impact on students' learning. This misalignment between the teaching approaches and the desired learning outcomes may explain the overall lack of evidence of the impact of research on teaching.

\section{Student Engagement and Learning}

While there is no evidence that research improves an academics' teaching, there is a strong body of evidence that students' learning is enhanced through their involvement in research. Students who undertake research in their undergraduate studies demonstrate improved retention and attainment, satisfaction and self-reported improvement in work readiness (Astin, 1994; cited in Prince, Felder, \& Brent, 2007). It is also argued that, with an adequate framework in place, research can improve student engagement and enhance the learning environment (Brew, 2010; Krause, Arkoudis, \& McCulloch, 2008). Brew (2010), reporting on a two year National Teaching Fellowship on enhancing undergraduate students' engagement through research states this "engages students meaningfully in higher education and prepares them for a 21st century world of work in which knowing how to create, inquire and critically evaluate knowledge is of increasing importance”. Creating a student experience in which there is integration of teaching and research also facilitates the induction of students into a community of learners outlined by the Boyer Commission. The Boyer commission's view is that a university is an ecosystem made up of communities of learners, and that teaching and learning should be explicitly linked within the environment (Boyer, 2000). The report recommends that universities build a culture that sees students as inquirers undertaking a journey of discovery. Higher education is seen as a site of exploration and adventure in which "every course in an undergraduate curriculum should provide an opportunity for a student to succeed through discovery-based methods" (Boyer, 2000). There is thus a strong imperative to inspire in students the passion and the capacity to generate and investigate important questions that impact on society. It is clear however, that there is a need to explore approaches to achieve this within higher education. In this paper we present a case study that outlines curriculum design and pedagogical strategies aimed at the integration of teaching and research to enhance student engagement and learning.

\section{Disciplinary Variation in the Teaching-Research Nexus}

There is increasing evidence that definitions and understandings of the teaching-research nexus varies across disciplines (Jenkins, 2004). This variation is in part determined by discipline-specific conceptualisations and practices of research, teaching and professional identity, and in the range of views regarding the nature of knowledge and ways in which knowledge is constructed. In the discipline of health sciences, the body of disciplinary knowledge is built up over time through peer reviewed research that critiques, builds on or extends the work of others. To participate in the community of researchers, a health science scholar must develop the tools of critical thinking and scientific analysis.

Engaging the student in the research process is one method to enhance the student's ability to critique and challenge current knowledge. In addition, promoting this link between teaching and research provides students with the opportunity to experience being part of a broader community of learners and researchers investigating a body of knowledge. There are clear advantages in integrating research and teaching within the discipline of food and nutrition. As Rowland reported, teaching in a discipline area that aims to develop a student's ability to "critique [of] matters of fundamental concern in the discipline" and "actively engage [ing] in research aimed at applying the discipline to a social or technical context” (Rowland, 1996) significantly enhances the integration of teaching and research. There are three ways to think of the interconnect between research and teaching in the University ecosystem: 1) expert knowledge passed onto the student (Neumann, 1994), 2) researcher and student learn together in a form of reciprocal relationship (Robertson \& Bond, 2001) and 3) students learning from the research culture of the laboratory or department (Brew \& Boud, 1995). In promoting a link between teaching and research, the students are being inducted into this community of practice and are provided with the awareness of and skills for the scholarship of discovery and application.

This paper follows the recommendations of the Boyer Commission whereby research is conceptualised as the Scholarship of Discovery (Boyer, 2000) with a focus on interpretative understanding of phenomena impacting on the population cohort. In addition, given that the case study outlined in this paper occurs within an undergraduate food and nutrition program with an applied nature, the research therefore may also be considered to encompass the Scholarship of Application (Boyer, 2000).

\section{Teaching Research}

The incorporation of research into the undergraduate curriculum should begin early to develop students' discipline-specific cognitive skills and establish an effective learning environment (Krause et al., 2008). The level of engagement of students in the learning process is a guide to the extent of learning that can be achieved, and an emphasis on research processes and problems may encourage engagement and stimulate learning. Learning can be enhanced by higher order thinking, and Anderson and Krathwohl (Anderson \& Krathwohl, 2001) extended Bloom's taxonomy to six levels of thinking that progress from a surface to a deeper approach: remembering; understanding; applying; analysing; evaluating; creating. In order to promote higher order thinking and therefore enhance learning, students 
must become active in the learning process, and one potential approach to encourage higher learning skills is to develop curriculum incorporating research which develops inquiry based learning.

\section{First Year Transition to Higher Education}

Students new to a university environment may have only experienced surface approaches to learning at secondary school, and the transition to deeper learning may be unfamiliar and therefore difficult for some university students. Indeed, one study found that two thirds of students surveyed believed secondary school did not did not prepare them for University learning environment (McInnis, James, \& Hartley, 2000). The university environment places more responsibility on the student to complete assessment tasks, readings, attend lectures and lab sessions independent of supervision. Those who adapt quickly to the new environment will thrive, while those who fail to adapt may struggle and become disengaged with the University learning environment. One third of first year students were not performing as well as they expected at University, and almost $50 \%$ of 1 st year students agreed that it was difficult to motivate themselves to study (McInnis et al., 2000). One of the issues for the teacher is to engage students early in the trimester, so they are motivated to undertake independent hours of study required to succeed.

The case study outlined in this paper guided food and nutrition students to undertake a research project involving the rigorous collection, analysis and evaluation of scientific data. The relevance and importance of the information was enhanced by designing a task in which students analysed their own diet or food behaviours and then reflected on why, when, where and with whom they eat. Thus the learning environment was immediately relevant to them. Given that a student's approach to learning will be determined by the learning context and assessment activity (Laurillard, 1997), this higher order thinking approach to conducting nutritional research is likely to increase learning quality (Anderson \& Krathwohl, 2001).

This paper outlines the integration of teaching and research within students' learning experience in a food and nutrition course. The aim of the project is to embed the development of research skills, knowledge and attitudes into the curriculum to enhance student engagement and learning. An experiential, inquiry based learning approach was implemented, in which students collected, analysed and interpreted research data using rigorous research methodology and an ethical and scientific approach

\section{Method}

First year University students enrolled in a Food and Nutrition course undertake the compulsory unit "Food: Nutrition, Culture and Innovation" in their first trimester. As this is one of the first units completed during their undergraduate degree, students' knowledge of food and nutrition is assumed to be at a level equivalent to that of a consumer. This unit was designed to provide a basic understanding of what and why we eat and implications for diet-related disease. As part of their assessment for this unit students are asked to complete three dietary research questionnaires with themselves as subjects. The questionnaires used in this assessment task are all frequently used in nutrition research and are of appropriate rigor for investigating food related behaviours and dietary intake. The aim of the assignment was to examine individual diet and food behaviours. Data collection was completed using systematic research techniques including 2 day diet diaries, a food behaviours questionnaire, and a food variety score questionnaire (Savige, HsuHage, \& Wahlqvist, 1997). During small group teaching sessions, each student was taught how to accurately complete the dietary analysis tools, with the tutors modelling the detail a researcher provides to a subject participating in research of this kind. This served two purposes, to demonstrate the proper methods for completion to ensure students developed a sound grasp of the process of administering dietary tools, and also to ensure the integrity of the data collected. The students then collected data on their own diet and eating related behaviours and additional questions were managed through the unit website on the University Learning Management System.

To complete the major assignment, students addressed fifteen questions specifically designed to foster an in-depth understanding of why they, as individuals, consume the foods they do. In order to answer these questions, students were required to analyse and interpret their own dietary and food behaviours data. The intended outcomes for students of this task were threefold: 1) to understand how to analyse and interpret dietary data 2) to gain self realisation about their own dietary habits, and 3) to provide an authentic the learning experience whereby students gain a practical understanding of the complexity of dietary research methodology, the problems that participants may experience when completing diet diaries, food variety scores and food behaviour questionnaires and the subsequent impact on data analysis and interpretation.

Sample questions from the Food Habits Assignment

Look at your answers for questions 14 - 22 (Food Behaviour Questionnaire). Of the answers you have given, please explain which ONE has the most influence on your diet. For example, does your ethnicity determine the type of food you eat, or you living arrangements, or your age etc.... Please explain your answer (100 words, 6 marks).

Do you think people who identify with a culture other than your own living in Australia would have similar eating patterns to you? Please explain (100 words, 6 marks).

The student assignments were graded and handed back to students, at which point students were also provided with a plain language statement and asked if they would consent to allowing their de-identified class data to be utilised for research purposes. Students were aware they could freely choose not to sign the consent form, in which case their data were not used in any research. This study was approved by the University's Human Research Ethics Committee.

Student evaluation of the unit Food: Nutrition, culture and innovation, was carried out by the Deakin University official “Student Evaluation of Teaching and Units' (SETU) process. All information entered by students was anonymous and group level quantitative data are publically available. Student comments were collated and these qualitative data are made available to the Chair of the unit after exam results are released. Release of the qualitative data for this publication has been approved by the University's Human Research Ethics Committee.

\section{Results and Discussion}

A total of 76 students of 118 (64\%) voluntarily completed the anonymous student evaluation of teaching units (SETU) at 
the completion of the unit. Table 1 shows results of the student evaluation survey of the unit. In general, the assignment and related pedagogy was well received by the majority of 1st year students. The data indicates that that the great majority of students agreed the assessment tasks were clear (88\%). This finding indicates that undertaking an assessment task that incorporates the learning and application of rigorous research methodologies did not result in any significant confusion or anxiety within the student cohort. While one student commented that "only one 2 hour tute needed for the assignment", the majority of student evaluations were favourable and the comments and data indicate most students were pleased to utilise the small group teaching to aide their understanding of the dietary tools and assignment.

"The fact that there was only the one major assignment was great. It was well managed by the staff and time was given to help students to understand" (SETU comment).

\section{Transition Pedagogy}

In general, in a university learning environment, many academic staff expect that students are self-motivated to complete the tasks they have been assigned. However, it is also important to acknowledge that the transition from secondary to tertiary education can be difficult one for many students. It was therefore important to ensure that students had maximum engagement with the topic, and the necessary scaffolding and support to develop the research skills required to complete the assignment effectively. In order to provide direction and support this transition to the university environment in which learning is more self-directed, a series of small group tutorials were held in the initial stages of the assignment. In these sessions students were instructed on how to complete the dietary information required, given examples of problems that may arise, and how to complete the questionnaires successfully. Students could go away and practice completing the forms to collect the data, then in the next small group meeting ask questions to assist them complete the assignment successfully.

Student Engagement and Authentic Learning

Student engagement and authentic learning are enhanced when the learning activity or assessment tasks are significant research questions or tasks that have genuine impact or value for the discipline or the individual involved (Bauer \& Bennett, 2003). In this case the data collected and analysed by the students; was determined to be of significant value to the discipline by providing insight into a unique, novel nutrition question as assessed via peer-review and publication in a leading nutrition research journal (Riddell, Ang, Keast, \& Hunter 2011). Furthermore, the authenticity of the learning was facilitated by using each individual as their own source of unique data. As every student selected the food and nutrition course, they are

Table 1.

Assessment of teaching in 1st year unit using 5-point scale.

\begin{tabular}{lcc}
\hline \multicolumn{1}{c}{ Question } & $\begin{array}{c}\text { 1-Strongly disagree } \\
\text { 5-Strongly agree }\end{array}$ & \% Agree \\
\hline The assessment tasks were clear & 4.3 & 88 \\
The teaching staff gave helpful feedback & 3.8 & 68 \\
The workload was manageable & 4.4 & 92 \\
Was this unit well taught & 4 & 74 \\
\hline
\end{tabular}

likely to have an inherent interest in food and nutrition, and each student has a set of unique food behaviours. During the data analysis phase of the assignment the students (also subjects) were encouraged to be reflective about their individual behaviours through a series of questions they answered. This approach provided a catalyst to inquiry as students could learn about their own dietary patterns and behaviours, which encouraged student engagement in the assignment (Kingsland, 1996).

"I just found the subject info interesting which really helped because then I wanted to do the assignments and study for it etc. I liked how it related to my life and I could take aspects and implement them into my every day life, which made it more interesting and effective" (SETU comment).

However, being required to complete an assignment that required introspection and good reflective practice was not appreciated by some students.

"I think the questions are too easy and based on personal experiences and opinions it is hard to mark fairly for each person. Also the word count was too restricted, trying to keep to the word count shouldn't be part of the challenge in getting a good mark, it should just be an indicator of how in depth you need to go" (SETU comment).

While this comment was isolated and did not appear to accurately reflect what the students in general thought of the assessment, it did raise an interesting point regarding feedback given, and as each student's answer to the questions would vary, consistent quality feedback was difficult to manage across the class as a whole. One student commented they did not like the feedback, although no additional detail was given. Table 1 shows a score of 3.8 with $68 \%$ of students agreeing feedback was helpful. The result was lower than expected, and indicates the need to improve this aspect of the assignment. The key criteria for feedback was to give credit when students exhibited sound reflective practice and analysis of their own dietary patterns, it did not matter if a diet consisted of hot dogs, beer, chips and chocolate. If the student could discuss their diet and behaviours in a logical reflective manner examining why they ate the way they did (more depth than "because I like it"), then the student would do well. It was not possible to give markers ideal answers for the questions, but they were provided with guidelines and example answers and the detail of feedback that students should expect.

The assignment was a major portion of the overall assessment of the unit, accounting for $40 \%$ of the final grade. Students were required to dedicate significant time to adequately complete the dietary tools and the subsequent analysis and interpretation. The significant time commitment required from students was matched by the significant weighting in terms of assessment proportion data. A large majority of students (92\%) believed that the workload was manageable. Data presented in Table 1 indicates that students were positive about the unit; furthermore the following comments reflect the general sentiment about the 1st year unit.

"It gave me an understanding of food as a whole new thing, rather than something that is consumed."

"I liked the prac, and also the assignment was very interesting."

"Incredibly important foundation subject to nutrition and understanding food choices."

"The food habits assignment was very interesting to record our diets" (SETU comments). 


\section{Academic Outcomes}

While it was a significant task, it was deemed successful and the framework is in place for subsequent years.

"Looking back I still find it hard to believe how successful the project has been. There is a natural fit between teaching and research and one which we can grow over the coming years" (Academic).

The results of this project integrating research into the undergraduate curriculum has enriched the research skills and knowledge of undergraduate students, and in addition served to induct them into the scientific community.

\section{Summary}

The aim of this project was to effectively integrate disciplenary teaching and learning with research. This integration was achieved through the combining of students' roles as both research subjects and students in an undergraduate unit. We were able to integrate students' participation in a systematic, rigorous data collection process required in a structured research environment with authentic learning of discipline specific skills and knowledge including dietary assessment and understanding why humans choose to eat the foods they do. Discipline specific integration of teaching and research were accomplished by aligning the learning outcomes with the learning activities associated with addressing a specific research question. Students were motivated and challenged by the process of participating actively in the discovery and generation of knowledge, rather than solely reading other's knowledge in journals or textbooks. The authenticity of the task was enhanced through the focus of the research being the students themselves, ensuring that both the research processes and the findings were particularly meaningful and relevant to the students. This authentic learning supported students' engagement with and attainment of research skills, culture and practice.

The majority of University academics have dual roles consisting of both teaching and maintaining an active research program. For many staff teaching at the undergraduate level, these roles remain quite distinct. Undergraduate students also experience this lack of integration of research practice in their learning, where their only research related activity is to search for and report on the findings of research conducted by others. The undergraduate learning experience outlined in this paper establishes an integrating experience whereby lecturers and students collaborate in authentic, discipline specific enquiry and knowledge creation.

\section{REFERENCES}

Anderson, L., \& Krathwohl, D. (Eds.) (2001). A taxonomy of learning, teaching, and assessment: A revision of Bloom's taxonomy of educational objectives. New York: Longman.

Bauer, K. W., \& Bennett, J. S. (2003). Alumni perceptions used to assess undergraduate research experience. The Journal of Higher Education, 74, 210-230. doi:10.1353/jhe.2003.0011

Boyer, E. (2000). Scholarship reconsidered: Priorities of the professsoriate: Princeton: The Carnegie Foundation for the Advancement of Teaching.

Brew, A. (2010) Enhancing undergraduate engagement through research and enquiry. URL (last checked 2 October 2012).

http://www.olt.gov.au/resource-enhancing-undergraduate-engagemen t-research-enquiry-macquarie-2010

Brew, A., \& Boud, D. (1995). Teaching and research: Establishing the vital link with learning. Higher Education, 29, 261-273. doi:10.1007/BF01384493

Coaldrake, P., \& Stedman, L. (1999) Academic work in the twenty-first century: Changing roles and policies. Canberra: Department of Education, Training and Youth Affairs Higher Education Division.

Feldman, K. (1987) Research productivity and scholarly accomplishment of college teachers as related to their instructional effectiveness: A review and exploration. Research in Higher Education, 26, 227298. doi:10.1007/BF00992241

Hattie, J., \& Marsh, H. (1996). The relationship between research and teaching-A meta-analysis. Review of Educational Research, 66, 507542.

Jenkins, A. (2004). A guide to the research evidence on teaching-research relations. Heslington: The Higher Education Academy.

Kingsland, A. (1996). Time expenditure, workload and student satisfaction in problem based learning. In L. Wilkerson, \& W. Gijselaers (Eds.), Bring problem based learning to higher education: Theory and practice (pp. 73-81). San Francisco: Jossey-Bass.

Krause, K., Arkoudis, S., \& McCulloch, R. (2008). The teaching-research nexus: A guide for academics and policy-makers in higher education. URL (last checked 2 October 2012). http://trnexus.edu.au/

Laurillard, D. (1997). Styles and approaches to problem solving. In F. Marton, D. Hounsell, \& N. Entwistle (Eds.), The experience of learning. Edinburgh: Scottish Academic Press.

McInnis, C., James, R., \& Hartley, R. (2000). Trends in the first year experience in Australian universities. Canberra: Department of Education, Training and Youth Affairs.

Neumann, R. (1994). The teaching-research nexus: Applying a framework to university students' learning experiences. European Journal of Education, 29, 323-340. doi:10.2307/1503744

Prince, M., Felder, R., \& Brent, R. (2007). Does faculty research improve undergraduate teaching? An analysis of existing and potential synergies. Journal of Engineering Education, 96, 283-294.

Riddell, L., Ang, B., Keast, R., \& Hunter, W. (2011) Impact of living arrangements and nationality on food habits and nutrient intakes in young adults. Appetite, 56, 726-731. doi:10.1016/j.appet.2011.02.010

Robertson, J., \& Bond, C. (2001). Experiences of the relation between teaching and research: What do academics value? Higher Education Research and Development, 20, 5-20. doi:10.1080/07924360120043612

Rowland, S. (1996). Relationship between teaching and research. Teaching in Higher Education, 1, 7-20. doi:10.1080/1356251960010102

Savige, G., Hsu-Hage, B., \& Wahlqvist, M. (1997). Food variety as a nutritional therapy. Current Therapeutics, 38, 57-67.

Zamorski, B. (2002) Research-led teaching and learning in higher education: A case. Teaching in Higher Education, 7, 411- 427. doi:10.1080/135625102760553919

Zubrick, A., Reid, I., \& Rossiter, P. (2001) Strengthening the nexus between teaching and research. Canberra: Department of Education, Training and Youth Affairs. 